\title{
Placement of ${ }^{131} \mathrm{Cs}$ permanent brachytherapy seeds in a large combined cavity of two resected brain metastases in one setting: case report and technical note
}

\author{
Jacelyn Greenwald, BA', Shoshana Taube, BA², Menachem Z. Yondorf, BA², Andrew Smith, MD², Albert Sabbas, PhD². \\ A. Gabriella Wernicke, MD, MSc,3 \\ ICornell University College of Arts and Sciences, Ithaca, New York, United States, 2Stich Radiation Oncology, Weill Cornell Medical College/ \\ New York-Presbyterian Hospital, New York, NY, United States, ${ }^{3}$ Department of Neurosurgery, Brain and Spine Center, Weill Cornell Medical \\ College/New York-Presbyterian Hospital, New York, NY, United States
}

\begin{abstract}
Large brain metastases are presently treated with surgical resection and adjuvant radiotherapy. However, local control (LC) for large tumors decreases from over $90 \%$ to as low as $40 \%$ as the tumor/cavity increases. Intraoperative brachytherapy is one of the focal radiotherapy techniques, which offers a convenient option of starting radiation therapy immediately after resection of the tumor and shows at least an equivalent LC to external techniques. Our center has pioneered this treatment with a novel FDA-cleared cesium-131 $\left({ }^{131} \mathrm{Cs}\right)$ radioisotope for the resected brain metastases, and published promising results of our prospective trial showing superior results from ${ }^{131} \mathrm{Cs}$ application to the large tumors $(90 \%)$. We report a 57-year-old male patient, with metastatic hypopharyngeal brain cancer. The patient presented with two metastases in the right frontal and right parietal lobes. Post-resection of these lesions resulted in a large total combined cavity diameter of $5.3 \mathrm{~cm}$, which was implanted with ${ }^{131} \mathrm{Cs}$ seeds. The patient tolerated the procedure well, with $100 \%$ local control and $0 \%$ radiation necrosis. This case is unique in demonstrating that the ${ }^{131} \mathrm{Cs}$ isotope was not only a convenient option of treating two resected brain metastases in one setting, but also that this treatment option offered excellent long-term LC and minimal toxicity rates.
\end{abstract}

Key words: cesium-131, ${ }^{131} \mathrm{Cs}$, brachytherapy, metastasis, radiation, radiotherapy.

\section{Purpose}

As advances in cancer treatment have extended life expectancy associated with cancer diagnosis, brain metastases have become increasingly common [1,2], and it is expected that $20-40 \%$ of cancer patients will develop brain metastases [2]. The current standard of care for these brain tumors includes radiotherapy techniques, surgical resection, or a combination of procedures. Whenever possible, surgery is still the preferred method for treating large brain metastases, but results with inadequate local control and recurrence rates nearing 50\% [3]. Recurrence rates can be reduced to $10-20 \%$ with the addition of post-operative whole brain radiation therapy (WBRT) $[4,5,6,7]$, but often with a detriment to quality of life (QoL) and an irreversible neurocognitive decline [8]. Hence, more focal techniques of radiotherapy have been utilized such as post-operative stereotactic radio- surgery (SRS) and intraoperative brachytherapy. Overall, these analyses have reported excellent local control rates. However, several studies have found a negative correlation between an increasing preoperative tumor size and a significantly diminished rate of local control. Preoperative tumor sizes of $>3.0 \mathrm{~cm}$ have been found to have reduced local control over a range of $40 \%$ to approximately $70 \%[9,10]$. This evidence suggests that SRS may not be the ideal adjuvant therapy for large resected metastases. Iodine-125 ( $\left.{ }^{125} \mathrm{I}\right)$ has offered the same local control as post-operative treatments such as SRS and WBRT [11,12]; however, the treatment with ${ }^{125} \mathrm{I}$ has induced long-term risk of high rates of radiation necrosis (up to 26\%) [11].

Our center at the Weill Cornell has pioneered the use of FDA-cleared novel cesium-131 $\left({ }^{131} \mathrm{Cs}\right)$ as a brachytherapy treatment in the setting of brain tumors. In a recently published prospective study of
Address for correspondence: A. Gabriella Wernicke, MD, MSc, Weill Medical College of Cornell University, Stich Radiation Oncology, 525 East $68^{\text {th }}$ Street, New York 10065, United States, phone: +1 212 746 3641, fax: +1 212746 8749, $₫$ e-mail: gaw9008@med.cornell.edu
Received: 14.02 .2019

Accepted: 02.07.2019

Published: 29.08.2019 
24 individuals treated with maximally safe neurological resection and intraoperative ${ }^{131} \mathrm{Cs}$ for the treatment of newly diagnosed brain metastases, there was a $100 \%$ rate of freedom from progression (FFP), with $0 \%$ incidence of radiation necrosis, determined clinically and radiographically (median follow-up of 19.3 months) [13]. However, a number of recent studies have shown that that local control is significantly reduced for resected tumors with large $(>3 \mathrm{~cm})$ preoperative diameters [14]. These studies suggest that a greater preoperative tumor diameter is a significant predictor of local failure [14]. In this report, we are presenting on a patient with two adjacent metastases, which were resected through one craniotomy. Based on the phase I and II trials and the previous cases with large metastases, we implanted one large cavity with ${ }^{131} \mathrm{C}$ s brachytherapy seeds. This report will analyze the impact of intraoperative ${ }^{131} \mathrm{Cs}$ brachytherapy employed in a tumor with a large preoperative tumor diameter $(5.34 \mathrm{~cm})$ and its impact on local control and radiation necrosis.

\section{Case report}

The patient, a 57-year-old male with a history of stage IV hypopharyngeal cancer, who was treated with chemotherapy and external beam radiation (70 Gy) to the neck in 2012. Two years later, the patient presented with a headache and was found to have a $1.5 \mathrm{~cm}$ left parietal lobe metastasis, which was treated with linac-based SRS to 20 Gy prescribed to an $80 \%$ isodose line on April $7^{\text {th }}$, 2015. Preoperative MRI on April $9^{\text {th }}, 2015$ revealed two metastases in the right frontal lobe and the right parietal lobe $1.9 \mathrm{~cm} \times 2.3 \mathrm{~cm} \times 2.4 \mathrm{~cm}$ and $2.7 \mathrm{~cm} \times 2.2 \mathrm{~cm}$ $\times 2.9 \mathrm{~cm}$, respectively (Figure 1). On April 10 $0^{\text {th }}, 2015$, the patient had both the right frontal and right parietal lesions treated with one right frontoparietal craniotomy and with the immediate intraoperative placement of ${ }^{131} \mathrm{Cs}$ brachytherapy. A gross total resection of both tu- mors was achieved, which combined to form one large cavity (over $5.3 \mathrm{~cm}$ total maximal preoperative tumor diameter). Minimal brain tissue was removed between the lesions, representing a total volume of approximately $0.5 \mathrm{~cm} \times 0.3 \mathrm{~cm} \times 0.4 \mathrm{~cm}$. A total of twenty-six ${ }^{131} \mathrm{Cs}$ stranded seeds with activity of $3.76 \mathrm{mCi}$ were implanted, with a dose of $80 \mathrm{~Gy}$ to a depth of $5 \mathrm{~mm}$ from the perimeter of the cavity, using the technique described in our original published phase I/II trial [13]. We used preoperative magnetic resonance imaging (MRI) scans as well as our institutional physics nomogram to calculate the dose of the volume implant. This was adjusted intraoperatively for the change in intracavitary volume after resection of the tumor. The $10 \mathrm{~cm}$, suture-stranded ${ }^{131} \mathrm{Cs}$ seeds $(0.5 \mathrm{~cm}$ inter-seed spacing) were delivered in strings of 10 seeds per string, subsequently cut into smaller lengths per the nomogram, and placed as a permanent volume implant along the cavity in a tangential pattern to maintain a 7 to $10 \mathrm{~mm}$ spacing between seeds. As a result, the combined cavity was lined with the seeds in a pattern like barrel staves or parallel tracks (Figure 2A). The ${ }^{131}$ Cs seeds were stranded and cut in real time in order to accommodate and precisely fit the resected cavity. The seeds were covered with surgicel (Ethicon) to prevent seed migration and alteration of dosimetry (Figure 2B), and tisseel (Baxter) was used to line the cavity to limit cavity shrinkage and further prevent seed dislodgement.

Within 24 hours post-implant, the patient underwent CT scanning to determine dose distribution. An insufficient implant is defined as the one with more than $10 \%$ of the area not covered by $90 \%$ of the prescribed dose. The dosimetry was excellent, and the patient did not require any additional radiation, as all areas were adequately covered.

Post-operative MRI revealed the gross total resection of both right frontal and right parietal tumors. The last follow-up visit on October 27th 2016 demonstrated the absence of tumor recurrence and no radiation necrosis
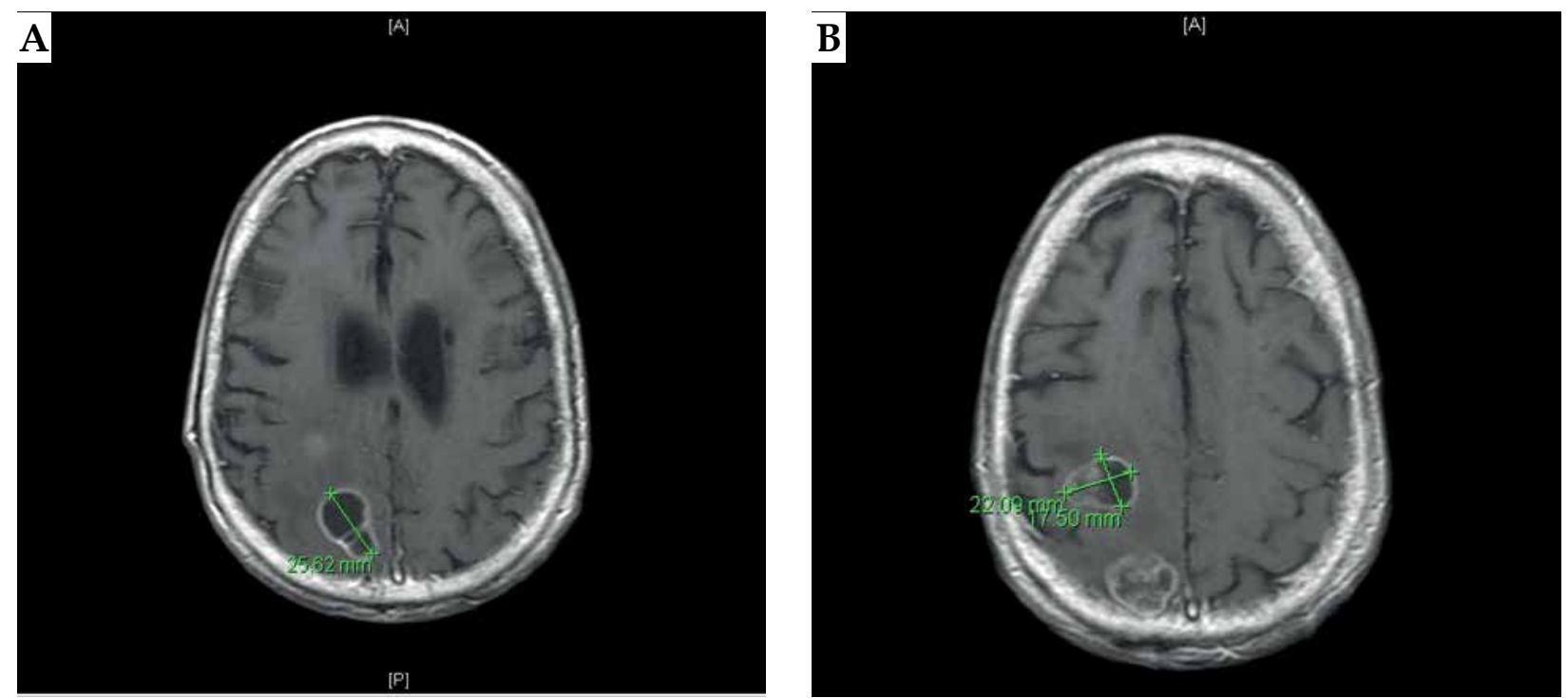

Fig. 1. A) Preoperative MRI of the brain: axial T1-weighted images demonstrate an enhancing anterior posterior right frontal lesion, measuring approximately $1.9 \mathrm{~cm} \times 2.3 \mathrm{~cm} \times 2.4 \mathrm{~cm}$. B) Preoperative MRI of the brain: axial T1-weighted images demonstrate an enhancing posterior right parietal lesion measuring approximately $2.7 \mathrm{~cm} \times 2.2 \mathrm{~cm} \times 2.9 \mathrm{~cm}$ 

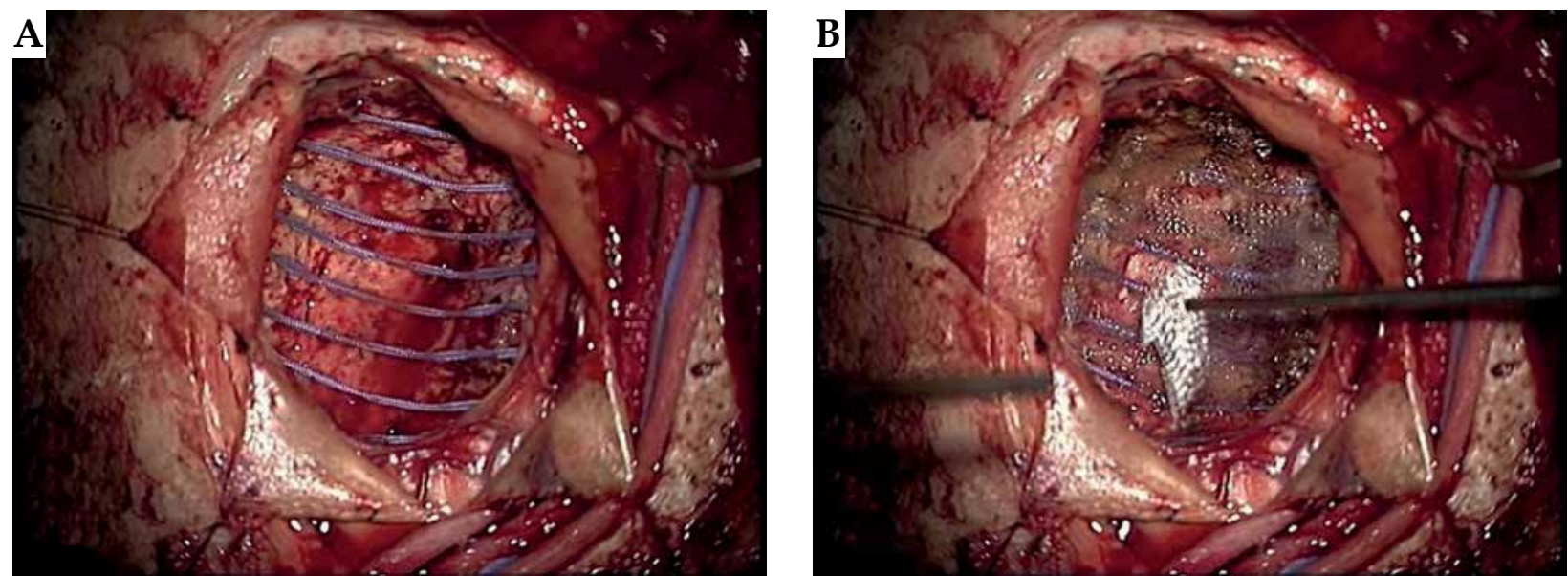

Fig. 2. A) Intraoperative photography demonstrating the resected cavity, intraoperative ${ }^{131} \mathrm{Cs}$ placement, and sealing the procedure: the combined cavity was lined with the seeds in a pattern like barrel staves or parallel tracks. B) Intraoperative photography demonstrating the resected cavity, intraoperative ${ }^{131} \mathrm{Cs}$ placement, and sealing the procedure: the seeds were then covered with surgicel (Ethicon) to prevent seed migration and alteration of dosimetry

(Figure 3). The patient excelled in the post-operative setting and had no neurological decline. Renal function precluded the patient from chemotherapy, and he remained disease-free for a significant period. His QoL was assessed before the procedure and at every follow-up, with questionnaires and mini-mental state examination (MMSE) as well as functional assessment of cancer therapy-brain (FACT-Br) questionnaire. The patient had an uneventful post-operative course and left a rehabilitation facility after 14 days. He developed a deep venous thrombosis (DVT) and subsequently a pulmonary embolism (PE), for which he was treated with anticoagulation. Eventually, the patient developed failure to thrive and was sent to a hospice, where he passed away one month later.

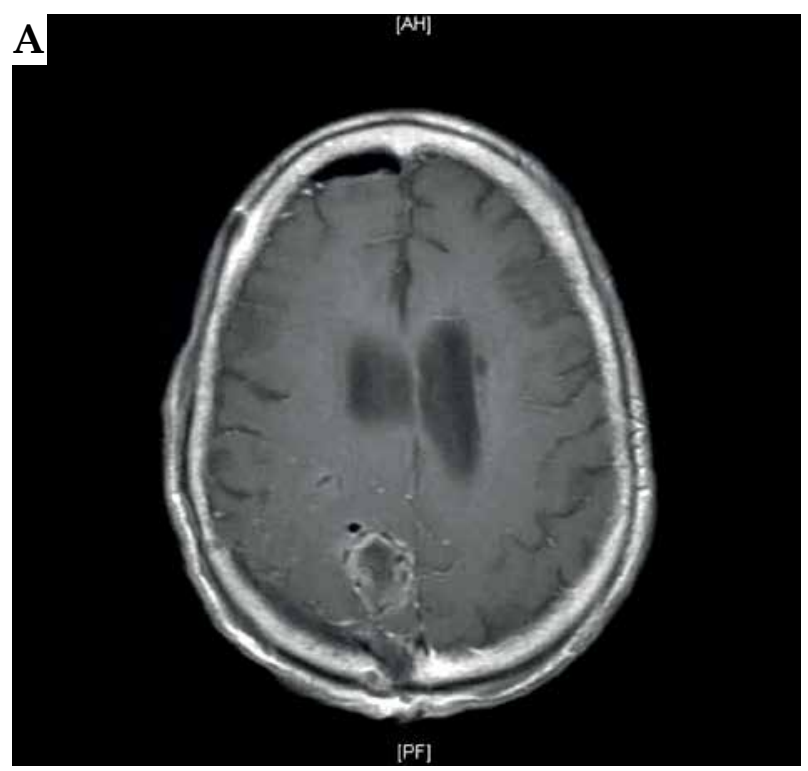

\section{Discussion}

The rate of local tumor recurrence in cancer patients after resection of a single brain metastasis is $25-40 \%$ [15]. Local techniques of radiotherapy, post-operative SRS, and intraoperative brachytherapy have been utilized in order to improve local control, yet spare patients from neurocognitive decline and diminished QoL associated with WBRT [16]. Current methods favor withholding WBRT until there are no alternative treatment options [17]. While post-operative SRS offers excellent LC rates in smaller brain metastases, several studies have found a large preoperative tumor diameter to be a significant predictor for local failure. In a study by Hartford et al.,

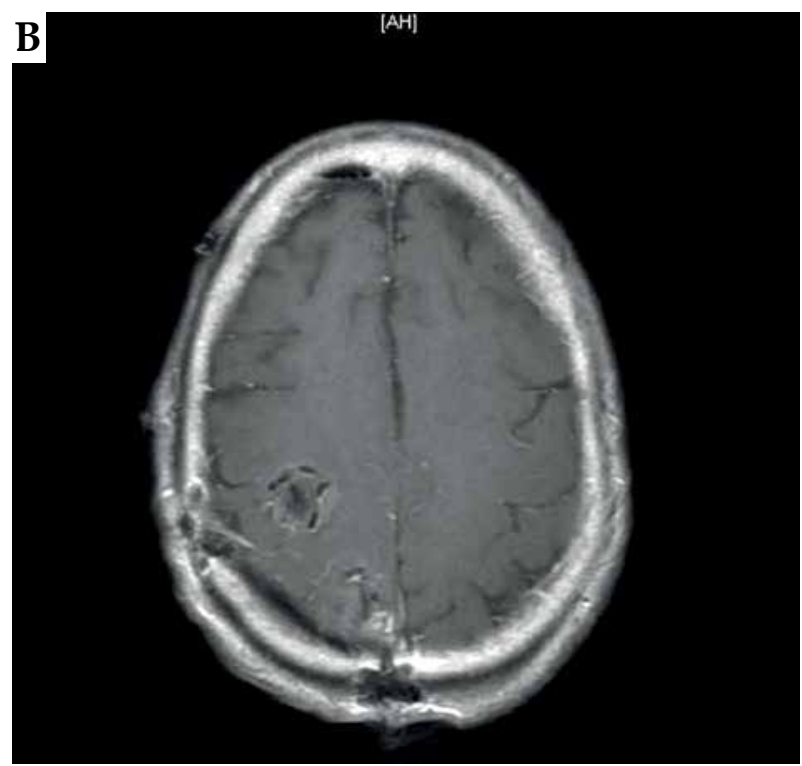

Fig. 3. A) Post-operative MRI of the brain after the right frontoparietal craniotomy demonstrating the intraoperative placement of ${ }^{131} \mathrm{Cs}$ brachytherapy seeds: axial T1-weighted images demonstrate an anterior posterior right frontal cavity with ${ }^{131} \mathrm{Cs}$ seeds. B) Post-operative MRI of the brain after the right frontoparietal craniotomy demonstrating the intraoperative placement of ${ }^{131} \mathrm{Cs}$ brachytherapy seeds: axial T1-weighted images demonstrate right parietal cavity implanted with ${ }^{131} \mathrm{Cs}$ seeds 
which evaluated brain metastases based on size, there was a 2-year actuarial local control rate of $89 \%$ for metastases $3.0 \mathrm{~cm}$ or smaller, versus only $40 \%$ for lesions larger than $3.0 \mathrm{~cm}(p<0.05)$. The only prospective study assessing tumor bed SRS is a phase II trial by Brennan et al. that also found increased local failure rates in resection cavities of tumors with preoperative diameters $>3 \mathrm{~cm}$ $(p=0.01)[9,10]$.

Brachytherapy offers an alternative procedure to external brain radiation therapy (EBRT) for the treatment of brain metastases. Through intraoperative radiation, it is possible to introduce immediate radiation treatment to the post-surgical cavity, thus eliminating the time between tumor resection and EBRT, when tumor regrowth is likely to occur. Permanent brachytherapy seeds are inserted along the surgical resection cavity and remain there permanently, preventing from future removal surgery. This is both cost-effective and associated with a decrease in the risk of infection or other surgical complications [18].

Brachytherapy seeds can be applied to a cavity of any size or shape. This is a tremendous advantage, as many SRS techniques are limited to smaller tumors of more round or spherical shapes. Furthermore, brachytherapy is valuable, because it lines the cavity and delivers radiation precisely to where the microscopic cancer cells may remain, unlike WBRT where the entire brain is subject to radiation increasing long-term risk of radiation necrosis or SRS, which delivers much of the radiation dose to an empty cavity [15]. Finally, brachytherapy offers a similar outcome to SRS and WBRT, with comparable local control for small tumors [11,12]. However, in this study, we hypothesize that brachytherapy is a good alternative treatment option to adjuvant post-operative SRS where, in large tumors, the rate of local control diminishes precipitously $(40-70 \%)[9,10]$.

For our patient, we chose to use ${ }^{131} \mathrm{Cs}$ radioisotope treatment, as it offers specific advantages over its historical predecessor ${ }^{125} \mathrm{I}$. In terms of dosimetry, the ${ }^{131} \mathrm{Cs}$ isotope was found to release a more homogeneous dosage in patients with prostate cancer, when compared to ${ }^{125}$ I or palladium-103 $\left({ }^{103} \mathrm{Pd}\right)$ prostate seed brachytherapy [19]. Compared to ${ }^{125} \mathrm{I},{ }^{131} \mathrm{Cs}$ has a higher dose-rate and a shorter half-life (9.7 days for ${ }^{131}$ Cs vs. 60 days for $\left.{ }^{125} \mathrm{I}\right)$ [15]. Because ${ }^{131}$ Cs delivers $90 \%$ of its total dosage within 33 days after implantation, the patient is free of radiation after one month, and can start a systemic therapy [15]. This contrasts with ${ }^{125} \mathrm{I}$, which delivers only $32 \%$ of the dose in one month and takes about 6 months of continuous brain tissue exposure to radiation for $100 \%$ dose delivery. This could be associated with high rates of radiation necrosis of up to $26 \%$, when treated with ${ }^{125}$ I [11]. Our recently published study revealed that ${ }^{131} \mathrm{Cs}$ rendered excellent local control with no incidences of radiation necrosis, as evidenced clinically and radiographically $[13,14]$. We have previously published a report on cavity shrinkage, where we determined that during the first month, when approximately $88 \%$ of ${ }^{131} \mathrm{Cs}$ dosage is delivered, there was an insignificant decrease in volume within the ${ }^{131} \mathrm{Cs}$ treatment group (median $22.0 \%, p=0.063$ ) [20].
We have published on QoL after ${ }^{131} \mathrm{Cs}$ implantation in a prospective study. Patients with brain metastasis who received intraoperative permanent ${ }^{131} \mathrm{Cs}$ brachytherapy implants saw an improvement of their neurocognitive status and self-assessment of QoL. We found that there was a statistical improvement in overall FACT-Br score at 4 and 6 months of follow-up when compared to baseline (162 vs. $143, p=0.004 ; 164$ vs. $143, p=0.005$, respectively), with an insignificant trend towards improvement at 2 and 12 months (154 vs. $143, p=0.067 ; 159$ vs. $149, p=0.4$, respectively). MMSE score was statistically improved at 4 months and up to 12 months compared to pre-treatment MMSE ( 30 vs. $29, p=0.017 ; 30$ vs. $29, p=0.001$, respectively) [21].

Several outcomes were measured during this case study. The first was freedom from progression (FFP). As of the last visit, on October 27, 2016, the MRI demonstrated no local recurrence of tumor to the right frontal or right parietal lobes, a representative of complete FFP. A second endpoint was toxicity or instance of radiation necrosis. Generally, radiation necrosis elevates with increasing dose size and size of the tumor lesion $[15,22]$. This is well understood with respect to ${ }^{125} \mathrm{I}$ isotope. However, no such toxicity is being seen with ${ }^{131} \mathrm{Cs}$ brachytherapy treatment, most likely due to its quick delivery (the consequence of a short half-life) and more compact dose distribution. Therefore, it is important to note that the patient presented with no adverse symptoms during follow-up and no signs of radiation necrosis on imaging.

The unique aspect of this case study is the size of the treated region. A large pre-treatment tumor diameter of $5.3 \mathrm{~cm}$ presents a large post-resection cavity for any adjuvant radiation technique. However, even in this case of a large treatment volume, ${ }^{131} \mathrm{Cs}$ application rendered excellent homogeneity and dose distribution, with $100 \%$ local control and $0 \%$ radiation necrosis. This suggests that ${ }^{131} \mathrm{Cs}$ brachytherapy could be successfully applied to a large tumors and combined resection cavities achieved through one craniotomy, although further investigation is needed. Given that this technique is entirely local and renders excellent homogeneity and coverage, we hypothesize that all respectable metastasis are candidates for this procedure. A prospective randomized trial will be able to set an upper limit on diameter or a volume of cavity as well as a long-term follow-up of toxicity profiles.

In conclusion, as modeled by this case study, brachytherapy using ${ }^{131} \mathrm{Cs}$ may offer an excellent treatment option for larger cavity volumes, with excellent local control and low toxicity. As the rates of local control diminish with the use of post-operative SRS in large resection cavities, our patient with a preoperative diameter of $5.3 \mathrm{~cm}$ sustained excellent results when treated using ${ }^{131} \mathrm{Cs}$ radioactive isotopes. A prospective study comparing intraoperative ${ }^{131} \mathrm{Cs}$ brachytherapy vs. post-operative SRS in large $(>3.0 \mathrm{~cm})$ brain metastases is gearing up at our institution.

\section{Disclosure}

The authors report no conflict of interest. 


\section{References}

1. Metellus P, Bialecki E, Le Rhun E, Dhermain F. Neurosurgical and radiosurgical decision making in brain metastasis patients in the area of targeted therapies. Chin Clin Oncol 2015; 4: 19

2. Patchell RA. The management of brain metastases. Cancer Treat Rev 2003; 29: 533-540.

3. Aoyama H, Shirato H, Tago M et al. Stereotactic radiosurgery plus whole-brain radiation therapy vs stereotactic radiosurgery alone for treatment of brain metastases. JAMA 2006; 295: 2483-2491.

4. Gaspar LE, Mehta MP, Patchell RA et al. The role of whole brain radiation therapy in the management of newly diagnosed brain metastases a systematic review and evidence-based clinical practice guideline. J Neurooncol 2010; 96: 17-32.

5. Hart MG, Grant R, Walker M, Dickinson H. Surgical resection and whole brain radiation therapy versus whole brain radiation therapy alone for single brain metastases. Cochrane Database Syst Rev 2005; (1): CD003292.

6. Kocher M, Soffietti R, Abacioglu U et al. Adjuvant wholebrain radiotherapy versus observation after radiosurgery or surgical resection of one to three cerebral metastases results of the EORTC 22952-26001 study. J Clin Oncol 2011; 29: 134141.

7. Patel KR, Prabhu RS, Kandula S et al. Intracranial control and radiographic changes with adjuvant radiation therapy for resected brain metastases: whole brain radiotherapy versus stereotactic radiosurgery alone. J Neurooncol 2014; 120: 657663.

8. Dagnew E, Kanski J, McDermott MW et al. Management of newly diagnosed single brain metastasis using resection and permanent iodine-125 seeds without initial whole-brain radiotherapy a two institution experience. Neurosurg Focus 2007; 22: E3.

9. Brennan C, Yang J, Hilden P et al. A phase 2 trial of stereotactic radiosurgery boost after surgical resection for brain metastases. Int J Radiat Oncol Biol Phys 2014; 88: 130-136.

10. Hartford AC, Paravati AJ, Spire WJ et al. Postoperative stereotactic radiosurgery without whole-brain radiation therapy for brain metastases Potential role of preoperative tumor size. Int J Radiat Oncol Biol Phys 2013; 85: 650-655.

11. Huang K, Sneed PK, Kunwar S et al. Surgical resection and permanent iodine-125 brachytherapy for brain metastases. J Neurooncol 2009; 91: 83-93.

12. Rogers LR, Rock JP, Sills AK et al. Results of a phase II trial of the GliaSite radiation therapy system for the treatment of newly diagnosed, resected single brain metastases. J Neurosurg 2006; 105: 375-384.

13. Wernicke AG, Yondorf MZ, Peng L et al. Phase III study of resection and intraoperative cesium-131 radioisotope brachytherapy in patients with newly diagnosed brain metastases. J Neurosurg 2014; 121: 338-348.

14. Wernicke AG, Hirschfeld CB, Smith AW et al. Clinical outcomes of large brain metastases treated with neurosurgical resection and intraoperative Cesium-131 brachytherapy results of a prospective trial. Int J Radiat Oncol Biol Phys 2017; 98: 1059-1068.

15. Wernicke AG, Schwartz T. [cdowns 1111] (2012, Dec 4). Metastatic utilizing Cesium-131 (Cs-131) graded seeds 2012/12 [Video file]. Retrieved from https://www.youtube.com/ watch?v=eor8gaXQKSE

16. Arvold ND, Lee EQ, Mehta MP et al. Updates in the management of brain metastases. Neuro Oncol 2016; 18: 1043-1065.

17. Higuchi $Y$, Yamamoto $M$, Serizawa $T$ et al. Modern management for brain metastasis patients using stereotactic ra- diosurgery literature review and the authors' gamma knife treatment experiences. Cancer Manag Res 2018; 10: 1889-1899.

18. Schulder M, Black PM, Shrieve DC et al. Permanent lowactivity iodine-125 implants for cerebral metastases. J Neurooncol 1997; 33: 213-221.

19. Yaparpalvi R, Rossinow J, Cavanagh WA et al. Is Cs-131 or I-125 or Pd-103 the "ideal" isotope for prostate boost brachytherapy - A dosimetric view point. Int J Radiat Oncol Biol Physics 2007; 69: S677-S678.

20. Wernicke AG, Lazow SP, Taube $S$ et al. Surgical technique and clinically relevant cavity dynamics following implantation of Cesium-131 brachytherapy in patients with brain metastases. Oper Neurosurg 2016; 12: 49-60.

21. Pham A, Yondorf MZ, Parashar B et al. Neurocognitive function and quality of life in patients with newly diagnosed brain metastasis after treatment with intra-operative cesium-131 brachytherapy a prospective trial. J Neurooncol 2016; 127: 63-71.

22. Shah $\mathrm{R}$, Vattoth $\mathrm{S}$, Jacob $\mathrm{R}$ et al. Radiation necrosis in the brain imaging features and differentiation from tumor recurrence. RadioGraphics 2012; 32: 1343-1359. 